\title{
Prevalence and Correlates of Fatigue in Chronic Kidney Disease and End-Stage Renal Disease: Are Sleep Disorders a Key to Understanding Fatigue?
}

\author{
Manisha Jhamb ${ }^{a}$ Kelly Liang ${ }^{a}$ Jonathan Yabes ${ }^{b}$ Jennifer L. Steel ${ }^{c, e}$ \\ Mary Amanda Dew ${ }^{c, d}$ Nirav Shah ${ }^{a}$ Mark Unruh $^{f}$ \\ ${ }^{a}$ Renal-Electrolyte Division, Department of Medicine, University of Pittsburgh, Departments of ${ }^{b}$ Medicine, \\ 'Psychiatry and d Psychology, Epidemiology and Biostatistics, University of Pittsburgh Medical Center, \\ e Department of Surgery, University of Pittsburgh School of Medicine, Pittsburgh, Pa., and ${ }^{\mathrm{f}}$ Nephrology Division, \\ Department of Medicine, University of New Mexico, Albuquerque, N.Mex., USA
}

\section{Key Words}

End-stage renal disease · Fatigue · Sleep · Chronic kidney disease $\cdot$ FACIT-F scale

\begin{abstract}
Background: Fatigue is an important symptom to patients with advanced chronic kidney disease (CKD). The aim of this study is to examine the prevalence and severity of fatigue among non-dialysis-dependent CKD and end-stage renal disease (ESRD) patients, to examine the association of fatigue with subjective and objective sleep quality, and to identify other modifiable factors associated with fatigue. Methods: A cross-sectional survey of 87 non-dialysis-dependent CKD (eGFR $\leq 45 \mathrm{ml} / \mathrm{min} / 1.73 \mathrm{~m}^{2}$ ) and 86 ESRD patients was done using the Functional Assessment of Chronic Illness Therapy - Fatigue (FACIT-F) and 36-Item Short-Form (SF-36) vitality scale. Higher FACIT-F score denoted less fatigue. Objective sleep was assessed using in-home polysomnography. Predictors of fatigue were determined using a linear regression model. Results: The mean FACIT-F score among all participants was $34.5 \pm 11.0$. Mean scores were similar among CKD and ESRD groups ( $34.25 \pm 11.28$ vs. $34.73 \pm 10.86 ; p=$
\end{abstract}

0.73). On univariate analyses, patients with higher levels of fatigue were more likely to have cardiovascular disease, benzodiazepine use, depressive symptoms, and slightly lower hemoglobin and serum albumin levels. There was no significant association between severity of sleep apnea and level of fatigue (Apnea Hypopnea Index $20.1 \pm 27.6$ vs. $20.3 \pm 22.0$; $\mathrm{p}=0.69$ ). Presence of cardiovascular disease, low serum albumin, depressive symptoms, poor subjective sleep quality, excessive daytime sleepiness and restless legs syndrome were independently associated with greater fatigue in multivariable regression models. The FACIT-F score correlated closely with the SF-36 vitality score $(r=0.81, p<0.0001)$. Conclusions: Patients with advanced CKD and ESRD experience profound fatigue. Depressive symptoms, restless legs syndrome, excessive daytime sleepiness, and low albumin levels may provide targets for interventions to improve fatigue in patients with advanced CKD. @ $2013 \mathrm{~s}$. Karger AG, Basel

An abstract based on the content of this article was presented as a poster at the 2011 American Society of Nephrology Kidney Week and 2013 World Congress of Nephrology Annual Meeting.

\section{KARGER}

E-Mail karger@karger.com

www.karger.com/ajn
(C) 2013 S. Karger AG, Basel

0250-8095/13/0386-0489\$38.00/0
Manisha Jhamb, MD, MPH

University of Pittsburgh School of Medicine

200 Lothrop Street, PUH C-1103

Pittsburgh, PA 15213 (USA)

E-Mail jhambm@upmc.edu 


\section{Introduction}

There is increasing awareness in the renal community of the high symptom burden and impaired health-related quality of life (HRQOL) experienced by patients with advanced chronic kidney disease (CKD). There has also been a recent shift in focus in research and patient-reported outcomes are being increasingly recognized as important outcome measures for large-scale trials such as the Frequent Hemodialysis Network trial [1]. More recently, patient-reported outcomes including fatigue have been advocated as important targets for individualization of anemia treatment in CKD patients [2]. Of the myriad of symptoms experienced by $\mathrm{CKD}$ and end-stage renal disease (ESRD) patients, fatigue is one of the most frequently reported symptoms. It affects $60-97 \%$ patients on long-term renal replacement therapy and up to $84 \%$ of CKD stage 5 patients $[3,4]$. Although there has been growing literature describing the prevalence and severity of fatigue in the ESRD population, studies evaluating fatigue among patients with non-dialysis-dependent CKD have been sparse and are limited by small sample size and a select population [3,5-7]. Moreover, there are no large studies comparing the severity of fatigue among non-dialysis-dependent CKD and ESRD population.

To develop interventions targeting fatigue in this population, understanding correlates and predictors of fatigue is needed first and foremost. One such modifiable correlate may be sleep disorders. Patients with advanced CKD and ESRD frequently have severe sleep-disordered breathing, restless legs, and excessive daytime sleepiness [8]. Poor self-reported sleep quality has been associated with fatigue in both hemodialysis (HD) and peritoneal dialysis (PD) patients [9-11]. However, there is little evidence on the association of objective sleep measures and fatigue. If such an association between sleep disorders [such as sleep apnea or restless legs syndrome (RLS)] and fatigue exists, then targeting interventions to treat the underlying sleep disorder may greatly improve energy levels and reduce fatigue in these patients.

The aim of the present study was to examine the prevalence and severity of fatigue among a cohort of advanced non-dialysis-dependent CKD (eGFR $\left.\leq 45 \mathrm{ml} / \mathrm{min} / 1.73 \mathrm{~m}^{2}\right)$ and ESRD patients. We assessed the severity of fatigue using two validated questionnaires - the Functional Assessment of Chronic Illness Therapy - Fatigue (FACIT-F) and the 36-Item Short-Form (SF-36). Further, we assessed the extent to which objective and subjective sleep disorders and quality, depressive symptoms and other modifiable factors were associated with fatigue in this patient population.

\section{Methods}

\section{Study Participants}

As part of a previously described [10] larger prospective cohort study investigating memory, sleep, and quality of life in advanced CKD patients, $87 \mathrm{CKD}$ and $86 \mathrm{ESRD}$ (17 PD and $69 \mathrm{HD}$ ) patients were enrolled from outpatient nephrology clinics, local dialysis centers, and kidney transplantation clinics in Western Pennsylvania between March 2004 and December 2008. Eligibility criteria was age $>18$ years and presence of advanced CKD (MDRD-derived eGFR $\leq 45 \mathrm{ml} / \mathrm{min} / 1.73 \mathrm{~m}^{2}$ ). Exclusion criteria included age $>90$ years, active medical disease (malignancy, myocardial infarction, advanced cirrhosis, advanced dementia), active alcohol abuse, refractory psychiatric disease. In addition, patients who were using continuous positive airway pressure, oral devices, or home oxygen therapy and those who had a tracheostomy were excluded. This study was approved by the University of Pittsburgh Institutional Review Board, and all participants provided written informed consent.

\section{Data Collection}

Baseline data collection included a brief standardized health interview, questionnaire administration, assessment of antidepressant and benzodiazepine use, and unattended home polysomnography (PSG). Age, sex, race, education, employment, alcohol use, and smoking status were self-reported. Additionally, standard serum laboratory tests drawn $\leq 3$ months prior to or following the PSG completion date were abstracted from the medical record.

\section{Self-Reported Fatigue Assessment}

Fatigue was assessed using the FACIT-F questionnaire, a 13item scale that assesses fatigue over the last 7 days using a 5-point Likert scale ranging from 'Not at all' to 'Very much'. It covers physical fatigue (e.g. I feel tired), functional fatigue (e.g. trouble finishing things), emotional fatigue (e.g. frustration), and social consequences of fatigue (e.g. limits social activity). Scores range from 0 to 52 with higher score indicating lower level of fatigue. The scale has been shown to have excellent internal consistency and testretest reliability $[12,13]$. For this study, we compared FACIT-F scores in our population to those in cancer patients with and without anemia (Hb 9.3 vs. $13.5 \mathrm{~g} / \mathrm{dl}$ ) based on a study by Cella et al. [14]. We divided the subjects into two groups (FACIT-F scores $>24$ vs. $\leq 24)$ based on the mean score among anemic cancer patients [14].

We also assessed fatigue using the vitality subscale of the widely used HRQOL instrument - SF-36 questionnaire. The vitality subscale consists of 4 items asking about energy, pep, and tiredness; scores range from 0 to 100 with higher scores reflecting greater energy and lower levels of fatigue [15].

Assessment of Self-Reported Sleep, Depression, and HRQOL

Participants were administered the Pittsburgh Sleep Quality Index (PSQI) questionnaire which includes 19 questions regarding habitual sleep over the past month. Higher PSQI scores reflect poorer sleep quality [16]. Participants also completed the Epworth Sleepiness Scale (ESS), an 8-item measure of likelihood of falling asleep in specific situations. Scores $\geq 10$ reflected excessive daytime sleepiness [17]. We used the Hopkins Restless Legs Syndrome Diagnostic Questionnaire, a series of 9 questions to establish essential criteria for RLS [18]. Depression was assessed using Patient Health 
Questionnaire (PHQ-9), a 9-item scale which asks about presence and severity of depressive symptoms within the last 2 weeks [19]. The SF-36 questionnaire was used to assess HRQOL [15].

Objective Sleep Assessment - Polysomnography

Unattended in-home PSG was performed using an ambulatory Compumedics Siesta monitor (Charlotte, N.C., USA) at habitual sleep times for one night. The PSG montage included bilateral central and occipital electroencephalogram channels $\left(\mathrm{C}_{3}-\mathrm{P}_{3}, \mathrm{C}_{4}-\mathrm{P}_{4}\right.$ and $\mathrm{C}_{\mathrm{Z}}-\mathrm{P}_{\mathrm{Z}}$ ), bilateral electrooculogram, and bipolar submentalis electromyogram. Bipolar electrocardiogram and position sensors were used to monitor heart rate and body position, respectively. Participants were also monitored for respiratory parameters, nasal pressure, and for abdominal and thoracic effort using finger pulse oximetry (Nonin, Minneapolis, Minn., USA), nasal-oral thermocouple, and inductance plethysmography, respectively. Trained PSG technologists scored sleep records for all study groups according to the guidelines of Rechtschaffen and Kales [20] using standard sleep stage scoring criteria for each 20 -second epoch. All scorers were blinded to the renal function of the patients. Standard definitions were used to identify apneas and hypopneas [21]; oximetry readings were used to quantify average and minimum oxyhemoglobin saturation levels and to quantify severity of nocturnal hypoxemia [21].

PSG outcome variables in the analysis included total sleep time (TST; sleep time excluding periods of wakefulness during the night), sleep efficiency (\% of TST/total of time spent attempting sleep), parameters of sleep architecture [\% of TST spent in stage 1, stage 2, stages 3-4, and rapid eye movement (REM) sleep], apnea/ hypopnea index (AHI; number of apneas and hypopneas/hour of sleep), periodic leg movement (PLM), and nocturnal hypoxemia ( $\geq 3 \%$ of TST with oxyhemoglobin saturation $<90 \%$ ). Severe sleep apnea was defined as having AHI $\geq 30$.

\section{Statistical Analyses}

Patient demographics, laboratory values and sleep measures were described using means and standard deviations (SD) for continuous variables and percentages for categorical variables. Differences between fatigue levels were assessed using Wilcoxon rank sum test for continuous variables and Fisher's exact test for categorical variables. Transformations were investigated and used for highly skewed distributions prior to model fitting. Univariable regression models were fitted to determine unadjusted associations with fatigue. Those that were significant at the $10 \%(\mathrm{p}<0.10)$ level were included one by one in a multivariable regression model that adjusted for age, sex, race, group (CKD vs. ESRD), antidepressant use, and benzodiazepine use. We used a linear regression model using FACIT-F as a continuous variable to determine correlates of fatigue in the adjusted models. All other statistical tests considered a significant level of $\mathrm{p}<0.05$. Assumptions were checked using standard linear model residual diagnostics. We also performed sensitivity analysis and tested for predictors treating FACIT-F as a dichotomous variable using logistic regression. We examined our multiple regression models for evidence of multicollinearity using variance inflation factors and found no evidence of collinearity between antidepressant use and PHQ-9 score. Thus, antidepressant use was adjusted for in the models. Pearson correlation was used to assess correlation between SF-36 vitality score and FACIT-F scores. All analyses were performed using SAS, version 9.3 statistical software (SAS Institute, Inc., Cary, N.C., USA).

Fatigue and Sleep Disorders in CKD and ESRD
Table 1. Patients' characteristic by levels of fatigue participants

\begin{tabular}{|c|c|c|c|}
\hline Variable & $\begin{array}{l}\text { FACIT } \leq 24 \\
\text { group } \\
\text { (high fatigue) } \\
(\mathrm{n}=34)\end{array}$ & $\begin{array}{l}\text { FACIT }>24 \\
\text { group } \\
\text { (low fatigue) } \\
(\mathrm{n}=139)\end{array}$ & $\mathrm{p}$ value \\
\hline FACIT-F score $(n=173)$ & $17.5 \pm 5.3$ & $38.7 \pm 7.5$ & $<0.0001$ \\
\hline Age $(n=173)$ & $49.2 \pm 13.7$ & $53.5 \pm 14.7$ & 0.13 \\
\hline Male gender $(\mathrm{n}=173)$ & 67.6 & 65.5 & 1.00 \\
\hline Race, black $(\mathrm{n}=164)$ & 20.6 & 29.5 & 0.39 \\
\hline \multicolumn{4}{|l|}{ High school education } \\
\hline$(\mathrm{n}=173)$ & 91.2 & 88.5 & 1.00 \\
\hline Married $(n=164)$ & 50.0 & 60.6 & 0.32 \\
\hline Employed $(\mathrm{n}=164)$ & 31.3 & 32.6 & 1.00 \\
\hline $\operatorname{CVD}(\mathrm{n}=134)$ & 47.6 & 23.9 & 0.03 \\
\hline Diabetes $(n=143)$ & 33.3 & 41.2 & 0.50 \\
\hline $\operatorname{BMI}(\mathrm{n}=173)$ & $28.5 \pm 6.1$ & $27.9 \pm 5.2$ & 0.76 \\
\hline Smoking status & & & 0.24 \\
\hline Current $(\mathrm{n}=21)$ & 21.9 & 10.6 & \\
\hline Past $(n=61)$ & 31.3 & 38.6 & \\
\hline Never $(\mathrm{n}=82)$ & 46.9 & 50.8 & \\
\hline Current vascular access & & & 0.86 \\
\hline $\operatorname{TDC}(\mathrm{n}=11)$ & 11.1 & 16.7 & \\
\hline $\mathrm{AVF} / \mathrm{G}(\mathrm{n}=44)$ & 61.1 & 61.1 & \\
\hline $\mathrm{PD}(\mathrm{n}=17)$ & 27.8 & 22.2 & \\
\hline \multicolumn{4}{|l|}{ Transplant evaluation } \\
\hline$(\mathrm{n}=147)$ & 80.8 & 68.6 & 0.25 \\
\hline Antidepressant use $(\mathrm{n}=164)$ & 18.8 & 15.2 & 0.60 \\
\hline Benzodiazepine use $(n=161)$ & 18.8 & 6.2 & 0.04 \\
\hline Albumin, g/dl $(\mathrm{n}=127)$ & $3.5 \pm 0.7$ & $3.9 \pm 0.5$ & 0.01 \\
\hline PTH $(\mathrm{n}=83)^{\mathrm{a}}$ & $\begin{array}{l}282.0 \\
(89.0,376.0)\end{array}$ & $\begin{array}{l}194.5 \\
(88.0,371.0)\end{array}$ & 0.62 \\
\hline CKD/ESRD group & & & 0.85 \\
\hline $\mathrm{CKD}(\mathrm{n}=87)^{1}$ & 52.9 & 49.6 & \\
\hline $\operatorname{ESRD}(\mathrm{n}=86)$ & 47.1 & 50.4 & \\
\hline eGFR $(n=157)$ & $13.1 \pm 7.0$ & $14.0 \pm 8.3$ & 0.84 \\
\hline $\mathrm{Hb}, \mathrm{g} / \mathrm{dl}(\mathrm{n}=110)$ & $11.2 \pm 1.3$ & $12.0 \pm 1.4$ & 0.048 \\
\hline Phosphorus, mg/dl $(\mathrm{n}=102)$ & $5.2 \pm 1.0$ & $5.1 \pm 1.8$ & 0.50 \\
\hline PHQ-9 $(n=164)$ & $14.3 \pm 4.6$ & $9.0 \pm 3.4$ & $<0.0001$ \\
\hline
\end{tabular}

Values are mean \pm SD or $\%$. ${ }^{\text {a }}$ Medians (25th, 75th percentile) are shown.

\section{Results}

\section{Patient Characteristics by Levels of Fatigue}

The characteristics of patients by different levels of fatigue are shown in table 1 . The mean $( \pm S D)$ FACIT-F score among all participants was $34.5 \pm 11.0$ (range 5-52). There was no difference in the level of fatigue among CKD and ESRD groups $(34.3 \pm 11.3$ vs. $34.7 \pm 10.9 ; \mathrm{p}=$ 0.73 ). As shown in table 1, univariate analyses revealed patients with cardiovascular disease, benzodiazepine use, depressive symptoms (higher PHQ-9 score), and slightly 


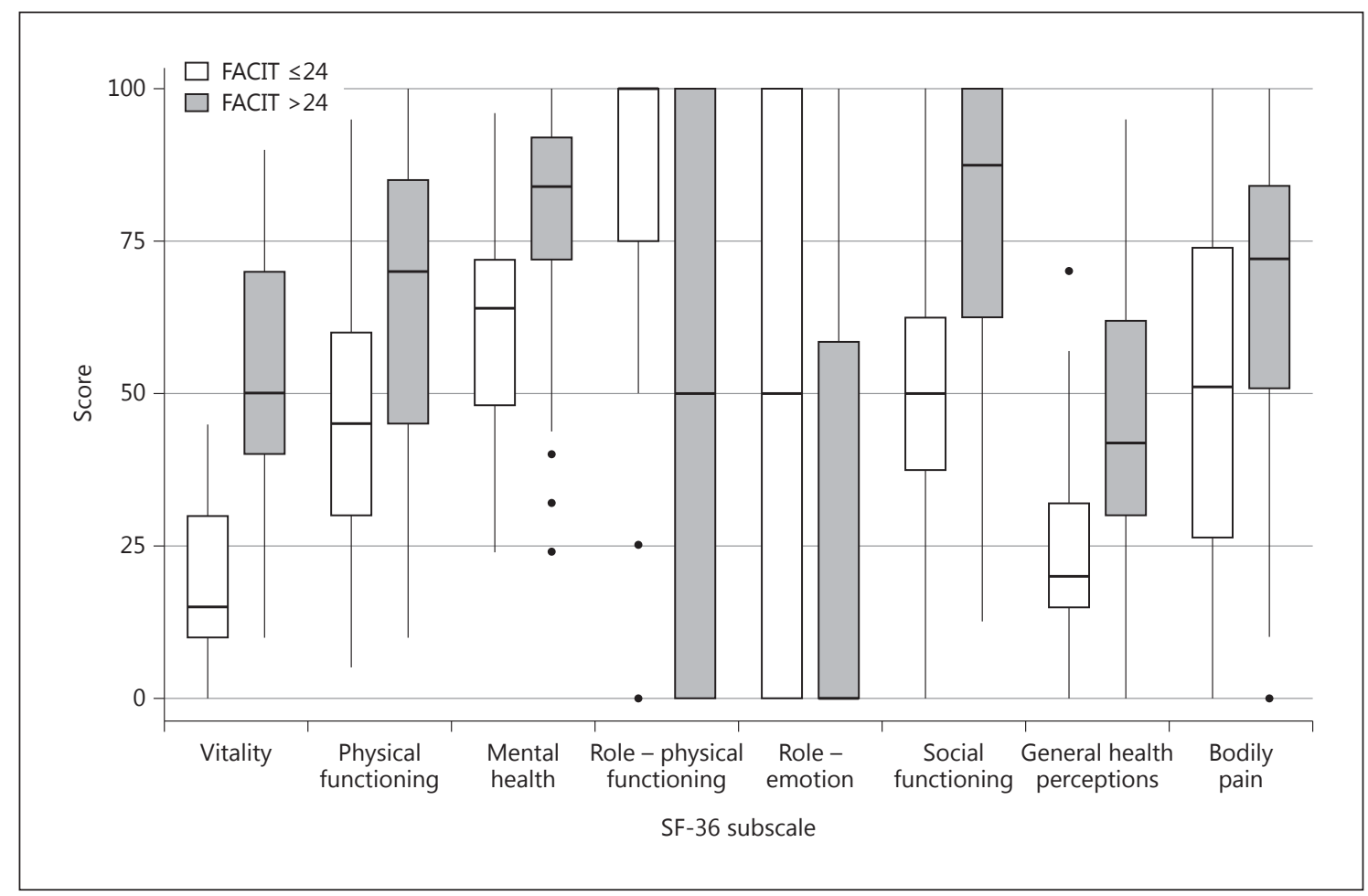

Fig. 1. HRQOL (measured by SF-36) by level of fatigue.

lower hemoglobin and serum albumin levels were more likely to experience higher levels of fatigue (lower FACITF score). There was no association of age, gender, race, diabetes or BMI with fatigue (table 1).

\section{HRQOL and Fatigue}

FACIT-F score correlated closely with the SF-36 vitality score $(\mathrm{r}=0.81, \mathrm{p}<0.0001)$. Those with worse fatigue as measured using the FACIT-F had significantly lower scores on SF-36 subscales of vitality, physical functioning, bodily pain, general health perceptions, social role functioning and mental health, but higher scores on physical role functioning and emotional role functioning ( $\mathrm{p}<$ 0.01) (fig. 1).

\section{Objective and Subjective Sleep Characteristics and \\ Fatigue}

Among the 173 enrolled participants, 93\% completed overnight at-home PSG. The average TST for the whole group was $342.9 \pm 111.0 \mathrm{~min}$ and did not vary significantly according to the level of fatigue $(\mathrm{p}=0.64)$. Longer duration of stage 2 sleep and nocturnal hypoxemia were significantly associated with greater fatigue on univariate analysis ( $\mathrm{p}=0.01$ and $\mathrm{p}=0.03$, respectively) (table 2 ). There was no significant difference in sleep efficiency, sleep latency, stages of sleep (except stage 2 sleep), AHI, PLM or REM sleep among subjects with higher versus lower FACIT-F scores (table 2). This remained true when we examined the association of objective sleep variables with FACIT-F as a continuous score (data not shown).

We found no significant relation of FACIT-F scores with the severity of sleep apnea (fig. 2). In our cohort, $72 \%$ of the patients had sleep apnea (AHI >5) and 22\% had severe sleep apnea (AHI $\geq 30)$. Among the $35(22 \%)$ subjects who were diagnosed as having severe sleep apnea $(\mathrm{AHI} \geq 30)$, the average FACIT-F score was $34.0 \pm 10.4$ versus $34.1 \pm 11.2$ in those with mild-moderate sleep apnea $(\mathrm{p}=0.93)$. In addition, no significant association between objective sleep and FACIT-F scores was observed on performing stratified analysis and looking at the CKD and ESRD group separately (data not shown). Our findings remained unchanged on performing sensitivity analysis using SF-36 vitality score.

On the other hand, poor subjective sleep quality was significantly associated with high levels of fatigue (table 2). Patients who reported poor sleep quality in the last 
Table 2. Subjective and objective sleep characteristics

\begin{tabular}{lccl}
\hline Variable & $\begin{array}{c}\text { FACIT } \leq 24 \\
\text { (high fatigue) }\end{array}$ & $\begin{array}{c}\text { FACIT }>24 \\
\text { (low fatigue) }\end{array}$ & $\begin{array}{l}\mathrm{p} \\
\text { value }\end{array}$ \\
\hline Total sleep time, min $(\mathrm{n}=161)$ & $341.4 \pm 127.8$ & $343.21 \pm 107.1$ & 0.64 \\
Sleep efficiency, \% $(\mathrm{n}=161)$ & $72.13 \pm 14.0$ & $72.0 \pm 15.9$ & 0.78 \\
Sleep latency, min $(\mathrm{n}=161)$ & $27.9 \pm 29.0$ & $39.7 \pm 53.3$ & 0.45 \\
\% stage 1 $(\mathrm{n}=161)$ & $10.3 \pm 5.9$ & $13.2 \pm 10.2$ & 0.19 \\
\% stage 2 $(\mathrm{n}=161)$ & $64.3 \pm 12.2$ & $58.8 \pm 11.7$ & 0.01 \\
\% stage 3-4 $(\mathrm{n}=161)$ & $7.6 \pm 8.4$ & $9.1 \pm 9.1$ & 0.45 \\
REM, \% ( $=161)$ & $17.9 \pm 10.3$ & $18.9 \pm 8.1$ & 0.53 \\
AHI ( $=161)$ & $20.1 \pm 27.6$ & $20.3 \pm 22.0$ & 0.69 \\
Nocturnal hypoxemia $(\mathrm{n}=143)$ & $10.7 \pm 13.5$ & $7.4 \pm 13.1$ & 0.03 \\
PLM ( $=159)$ & $3.8 \pm 4.2$ & $4.8 \pm 5.1$ & 0.32 \\
Arousals $(\mathrm{n}=157)$ & $21.9 \pm 8.3$ & $24.9 \pm 10.9$ & 0.24 \\
Wake after sleep onset $(\mathrm{n}=161)$ & $101.0 \pm 63.7$ & $94.0 \pm 56.2$ & 0.67 \\
PSQI ( $\mathrm{n}=135)$ & $9.8 \pm 4.1$ & $7.0 \pm 4.1$ & 0.003 \\
ESS ( $=157)$ & $10.2 \pm 5.6$ & $7.8 \pm 4.1$ & 0.03 \\
RLS (n=161) & 62.5 & 34.1 & 0.005 \\
\hline
\end{tabular}

Mean \pm SD or $\%$.

Table 3. Predictors of worse fatigue in a multivariable linear regression model ${ }^{\mathrm{a}}$

\begin{tabular}{lrlc}
\hline Characteristic & Beta $^{\mathrm{b}}$ & $\begin{array}{l}\text { Standard } \\
\text { error }\end{array}$ & $\begin{array}{l}\mathrm{p} \\
\text { value }\end{array}$ \\
\hline Cardiovascular disease & -5.48 & 2.15 & 0.01 \\
Albumin (log) & 21.09 & 5.89 & $<0.001$ \\
Depressive symptoms (log) & -18.25 & 1.81 & $<0.001$ \\
Self-reported sleep quality & -3.67 & 1.16 & 0.002 \\
Daytime sleepiness & -4.57 & 1.10 & $<0.001$ \\
Restless legs syndrome & -5.88 & 1.75 & $<0.001$ \\
\hline
\end{tabular}

a Adjusted for age, sex, race, group (CKD vs. ESRD), antidepressant use and benzodiazepine use.

${ }^{b}$ Expected change in FACIT-F score for each unit increase in the characteristic variables.

1 month (higher PSQI score) had excessive daytime sleepiness (higher ESS score) and had symptoms of RLS (higher Hopkins RLS score) had worse fatigue (lower FACIT-F score).

\section{Independent Predictors of Worse Fatigue}

As shown in table 3, we found that cardiovascular disease, low serum albumin, depressive symptoms, poor subjective sleep quality, excessive daytime sleepiness and RLS were independently associated with worse fatigue af-

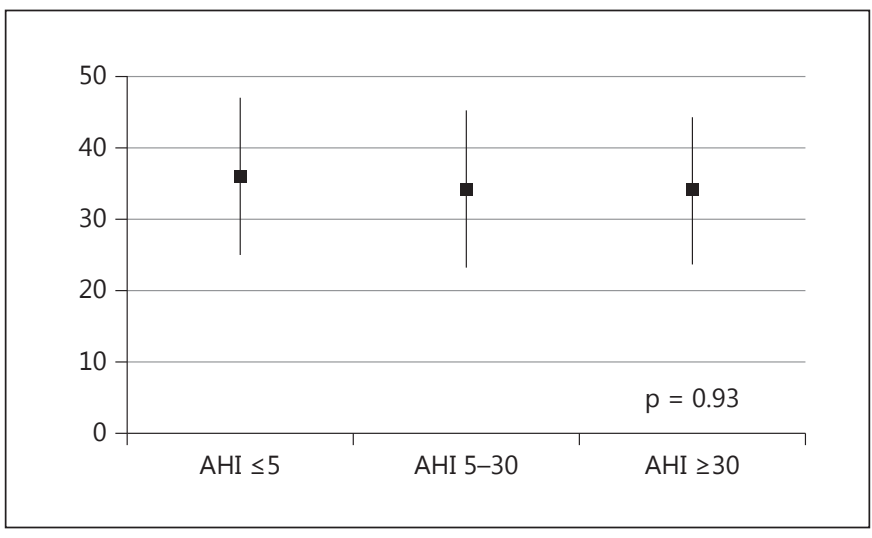

Fig. 2. FACIT scores by severity of sleep apnea. The black box represents the mean and the line represents the SD for the FACIT-F scores for each AHI subgroup.

ter adjusting for age, race, gender, group (CKD vs. ESRD), and medication use (benzodiazepines, antidepressants). Multivariable logistic regression treating FACIT-F as a dichotomous outcome was also performed as an alternative analytic approach and it revealed similar results.

\section{Discussion}

Our findings demonstrate that patients with advanced non-dialysis-dependent CKD and ESRD experience profound fatigue. The average fatigue level among these participants was substantially worse than the general population (FACIT-F score $34.5 \pm 11.0$ in CKD/ESRD vs. $43.6 \pm$ 9.4 in general population) [14]. In fact, $20 \%$ of the subjects had level of fatigue comparable to anemic cancer patients (FACIT-F score $23.9 \pm 12.6$ ) [14]. Interestingly, we observed that the severity of fatigue was similar in the nondialysis-dependent CKD and ESRD. We also identified some important correlates of fatigue, such as depressive symptoms, low albumin levels and RLS which may be targets for interventions to improve fatigue in this population. Lack of a significant association of fatigue with objective sleep-disordered breathing was striking and may suggest that these are distinct issues in this population.

The finding that patients with advanced CKD and ESRD experience similar severity of fatigue was counterintuitive. One would expect non-dialysis-dependent CKD patients to be more functional, physically active and have lesser fatigue than patients who undergo dialysis and have high level of dialysis-related fatigue [22]. One could posit that there may be different underlying etiological 
mechanisms contributing to fatigue in these subgroups. It may be that kidney disease-related factors such as uremia and pruritus that contribute to fatigue among nondialysis-dependent CKD are mitigated and replaced by treatment-related factors among dialysis-dependent ESRD patients.

Fifty-three percent of the patients in the study had depression as suggested by a PHQ- 9 score $\geq 10$. The finding that fatigue was positively associated with depressive symptoms even after adjusting for other variables including use of antidepressant medication extends previous findings [23, 24]. However, whether a causal mechanism exists between the two is uncertain. Future studies elucidating this intricate relationship and the direction of effects may help develop more effective mechanisms to address fatigue and depression in this population.

Consistent with our prior work in HD patients, we found that lower albumin levels were associated with worse fatigue $[23,25]$. We extended these findings in this study while accounting for the influence of depression, and by using both FACIT-F and SF-36. In addition, we extended this finding to patients with advanced CKD not on dialysis. Hypoalbuminemia, which is a marker of malnutrition-inflammation complex syndrome and a strong predictor of poor HRQOL and cardiovascular death [26-29], may also similarly affect fatigue in CKD and ESRD patients. Specific interventions aimed at improving nutritional status or increasing albumin levels by emerging pharmacological interventions such as anabolic steroids, appetite stimulants or anti-inflammatory therapies $[30,31]$ may perhaps have a beneficial effect on fatigue.

Sleep disorders have been hypothesized to cause fatigue through two separate mechanisms - by causing excessive daytime sleepiness and through biological mechanisms involving increased levels of certain inflammatory cytokines [31]. Our study revealed a significant association between fatigue and self-reported sleep quality, excessive daytime sleepiness and RLS symptoms. Surprisingly, we did not find any significant association between sleep-disordered breathing and fatigue, although a significant number of patients in our study had moderate to severe untreated sleep apnea. This remained true when we looked at the CKD and the ESRD group separately. A possible hypothesis may be that sleep disorders such as sleep apnea manifest primarily with sleepiness rather than fatigue and there are separate causes of fatigue in this advanced CKD/ESRD population. Alternatively, it may be that the effect of sleep apnea on fatigue is masked by other contributors to fatigue in this population. Further research is warranted to investigate the relationship between sleep disorders (such as insomnia, sleep-disordered breathing, short sleep duration), RLS and fatigue in the CKD and ESRD patients. To this end, we are conducting a clinical study - Blood pressure In Dialysis - Sleep (BID-SLEEP) - evaluating sleep apnea and fatigue among HD patients and impact of intensive blood pressure and volume control in these patients on sleep apnea and fatigue.

Our study has a number of strengths. We examined fatigue in a relatively large population of non-dialysisdependent CKD patients and compared the severity of fatigue with ESRD patients. Ours is the first study to investigate the association between objective sleep measures and fatigue in the CKD and ESRD population. In addition, we used standardized PSG assessments and scoring across study groups. Moreover, we used a validated fatigue questionnaire that characterizes the multidimensional aspect of fatigue better than the widely used SF-36 vitality scale. Lastly, we measured depressive symptoms using a validated depression questionnaire and examined its association with fatigue, unlike some of the prior large-scale studies of fatigue in ESRD patients which have used the SF-36 mental health scores $[9,25]$.

Our findings should be considered in light of several limitations. First, we used unattended in-home PSG. However, prior studies using home PSG have consistent findings from studies using full PSG performed in the sleep lab [32]. Second, we did not assess the association of other sleep disorders such as insomnia with fatigue, although PSQI captures insomnia to some extent. Also, the overnight sleep duration was measured by PSG, but we did not have data on daytime naps in these groups. Third, patients on conventional three times weekly HD may have significant day-to-day and diurnal variation in fatigue in relation to the timing of dialysis which is not captured by survey questionnaires. Future work should consider using ecological momentary assessment to incorporate real-time assessments to measure fatigue repeatedly and reliably [33]. In addition, since the PHQ-9 questionnaire includes items assessing fatigue (feeling tired or having little energy), we might be measuring conceptually overlapping constructs. Lastly, this study was cross-sectional, thus we cannot predict the direction of relationship between variables or causality [34].

In conclusion, the results of our study provide clinically relevant information on the prevalence and predictors of fatigue in the CKD and ESRD population. The study population of advanced CKD and ESRD patients 
demonstrated profound levels of fatigue. It is important to note that some of the correlates of fatigue such as depressive symptoms, low albumin level and RLS may be modifiable and amenable to interventions. Future studies looking at effect of anti-inflammatory interventions on fatigue and sleep disorders are needed.

\section{Acknowledgements}

This work was supported by NIH grant R01DK077785 (M.U.) and American Heart Association grant 11FTF7520014 (M.J.).

\section{Disclosure Statement}

The authors have no conflicts of interest to disclose.

\section{References}

$>_{1}$ Jhamb $\mathrm{M}$, et al: Design and rationale of health-related quality of life and patient-reported outcomes assessment in the Frequent Hemodialysis Network trials. Blood Purif 2011;31:151-158.

-2 Kliger AS, Fishbane S, Finkelstein FO: Erythropoietic stimulating agents and quality of a patient's life: individualizing anemia treatment. Clin J Am Soc Nephrol 2012;7:354-357.

3 Murtagh FE, et al: Symptoms in advanced renal disease: a cross-sectional survey of symptom prevalence in stage 5 chronic kidney disease managed without dialysis. J Palliat Med 2007;10:1266-1276.

-4 Murtagh FE, Addington-Hall J, Higginson IJ: The prevalence of symptoms in end-stage renal disease: a systematic review. Adv Chronic Kidney Dis 2007;14:82-99.

5 Bonner A, Wellard S, Caltabiano M: The impact of fatigue on daily activity in people with chronic kidney disease. J Clin Nurs 2010;19: 3006-3015.

6 Fukuhara S, et al: Understanding measurements of vitality in patients with chronic kidney disease: connecting a quality-of-life scale to daily activities. PLoS One 2012;7:e40455.

7 Macdonald JH, et al: Exertional fatigue in patients with CKD. Am J Kidney Dis 2012;60: 930-939.

8 Roumelioti ME, et al: Sleep-disordered breathing and excessive daytime sleepiness in chronic kidney disease and hemodialysis. Clin J Am Soc Nephrol 2011;6:986-994.

9 Jhamb M, et al: Correlates and outcomes of fatigue among incident dialysis patients. Clin J Am Soc Nephrol 2009;4:1779-1786.

10 Unruh ML, et al: Sleep quality and its correlates in the first year of dialysis. Clin J Am Soc Nephrol 2006;1:802-810.

11 Yngman-Uhlin P, Edell-Gustafsson U: Selfreported subjective sleep quality and fatigue in patients with peritoneal dialysis treatment at home. Int J Nurs Pract 2006;12:143-152.

12 Wadler S, et al: Randomized phase II trial of either fluorouracil, parenteral hydroxyurea, interferon- $\alpha_{2 a}$, and filgrastim or doxorubicin/ docetaxel in patients with advanced gastric cancer with quality-of-life assessment: Eastern Cooperative Oncology Group Study E6296. Cancer J 2002;8:282-286.
13 Yellen SB, et al: Measuring fatigue and other anemia-related symptoms with the Functional Assessment of Cancer Therapy (FACT) measurement system. J Pain Symptom Manage 1997;13:63-74.

14 Cella D, et al: Fatigue in cancer patients compared with fatigue in the general United States population. Cancer 2002;94:528-538.

15 DeOreo PB: Hemodialysis patient-assessed functional health status predicts continued survival, hospitalization, and dialysis-attendance compliance. Am J Kidney Dis 1997;30: 204-212.

16 Buysse DJ, et al: The Pittsburgh Sleep Quality Index: a new instrument for psychiatric practice and research. Psychiatry Res 1989;28: 193-213.

17 Johns MW: A new method for measuring daytime sleepiness: the Epworth Sleepiness Scale. Sleep 1991;14:540-545.

18 Allen RP, Earley CJ: Validation of the Johns Hopkins restless legs severity scale. Sleep Med 2001;2:239-242.

19 Kroenke K, Spitzer RL, Williams JB: The PHQ-9: validity of a brief depression severity measure. J Gen Intern Med 2001;16:606-613.

20 Rechtschaffen A, Kales A: A Manual of Standardized Terminology, Techniques, and Scoring System for Sleep Stages of Human Subjects. Bethesda, US Department of Health, Education, and Welfare Public Health Service - NIH/NIND. 1968.

21 Sleep-related breathing disorders in adults: recommendations for syndrome definition and measurement techniques in clinical research. The Report of an American Academy of Sleep Medicine Task Force. Sleep 1999;22: 667-689.

22 Caplin B, Kumar S, Davenport A: Patients' perspective of haemodialysis-associated symptoms. Nephrol Dial Transplant 2011;26: 2656-2663.

23 Bossola M, Luciani G, Tazza L: Fatigue and its correlates in chronic hemodialysis patients. Blood Purif 2009;28:245-252.
24 Karakan S, Sezer S, Ozdemir FN: Factors related to fatigue and subgroups of fatigue in patients with end-stage renal disease. Clin Nephrol 2011;76:358-364.

25 Jhamb M, et al: Impact of fatigue on outcomes in the hemodialysis (HEMO) study. Am J Nephrol 2011;33:515-523.

26 Herselman M, et al: Relationship between serum protein and mortality in adults on longterm hemodialysis: exhaustive review and meta-analysis. Nutrition 2010;26:10-32.

27 Rambod M, et al: Association of Malnutrition-Inflammation Score with quality of life and mortality in hemodialysis patients: a 5 -year prospective cohort study. Am J Kidney Dis 2009;53:298-309.

28 Kalantar-Zadeh K, et al: A malnutrition-inflammation score is correlated with morbidity and mortality in maintenance hemodialysis patients. Am J Kidney Dis 2001;38:1251-1263.

29 Kalantar-Zadeh K, et al: Comparing outcome predictability of markers of malnutrition-inflammation complex syndrome in haemodialysis patients. Nephrol Dial Transplant 2004; 19:1507-1519.

30 Ikizler TA, et al: Prevention and treatment of protein energy wasting in chronic kidney disease patients: a consensus statement by the International Society of Renal Nutrition and Metabolism. Kidney Int 2013 (E-pub ahead of print).

31 Kopple JD, et al: OPPORTUNITY: a randomized clinical trial of growth hormone on outcome in hemodialysis patients. Clin J Am Soc Nephrol 2008;3:1741-1751.

32 Jhamb M, et al: Fatigue in patients receiving maintenance dialysis: a review of definitions, measures, and contributing factors. Am J Kidney Dis 2008;52:353-365.

33 Flemons WW, et al: Home diagnosis of sleep apnea: a systematic review of the literature. An evidence review cosponsored by the American Academy of Sleep Medicine, the American College of Chest Physicians, and the American Thoracic Society. Chest 2003; 124:1543-1579.

34 Hacker ED, Ferrans CE: Ecological momentary assessment of fatigue in patients receiving intensive cancer therapy. J Pain Symptom Manage 2007;33:267-275. 